\title{
Search for dark matter by the Yakutsk array data
}

\author{
A.A. Mikhailov a and V.A. Kolosov \\ Yu.G. Shafer Institute of Cosmophysical Research and Aeronomy, 31 Lenin Ave., 677980 Yakutsk, Russia
}

\begin{abstract}
We analyzed the Yakutsk Extensive Air Shower array data registered from 1974 to 2011. 14 particles were found that come directly from the Galactic Center to Earth without any deflection in the Galactic magnetic field. We suppose that these are particles of dark matter.
\end{abstract}

\section{Introduction}

We analyzed the distribution of particles detected by the Yakutsk array for energies $E \geq 8.10^{18} \mathrm{eV}$ from 1974 until 2011. We considered the arrival directions of extensive air showers (EAS) with zenith angles $\theta<60^{\circ}$ and whose cores were situated inside the perimeter of the array. The energy of the particles was determined by registering Cherenkov light with an accuracy of $\sim 30 \%$ and for angles $\sim 5^{\circ}-7^{\circ}$.

\section{The distribution of particles in right ascension}

At first we analyzed the distribution of particles in right ascension by harmonic analyses for energies $E \geq$ $8.10^{18} \mathrm{eV}$. For these energies we found the amplitude and phase in right ascension of the $1^{\text {st }}$ harmonic of Fourier analysis to be $r_{1}=0.14 \pm 0.04, \phi_{1}=0.6^{\circ} \pm 19^{\circ}$, $\mathrm{r}_{2}=0.02 \pm 0.04, \phi_{2}=8^{\circ} \pm 125^{\circ}$. The total number of particles detected was 938 and the chance probability, $\mathrm{P}$, having amplitudes $r_{1} \geq 0.14$ is $\sim 0.01$.

Earlier we suggested a new method to analyze data only along two coordinates, declination and right ascension, and we analysed the data by this method [1]. We did not find any statistical significance in the amplitude of arrival directions of particles by Fourier analyses, but phases of the $1^{\text {st }}$ harmonic at energies $E>10^{19} \mathrm{eV}$ start to change at right ascension $\mathrm{RA} \sim 180^{\circ}$ or at high galactic latitudes, b (Fig. 1). Possibly this change of phase of the $1^{\text {st }}$ harmonic most likely points to a change of sources of ultrahigh energy cosmic rays to high latitudes.

\section{The distribution of particles with energies $E>8.10^{18} \mathrm{eV}$ in the Celestial sphere}

In a previous paper [1] we concluded that cosmic rays up to energy $E<4 \times 10^{19} \mathrm{eV}$ are galactic and their sources are pulsars. We have found 6 regions, including data from the Telescope Array (TA), with many pulsars: inside the $1^{\text {st }}$ region -39 pulsars, the $2^{\text {nd }}$ region -16 pulsars, the $3^{\text {rd }}$ region $->10$ pulsars

a e-mail: mikh@ikfia.ysn.ru

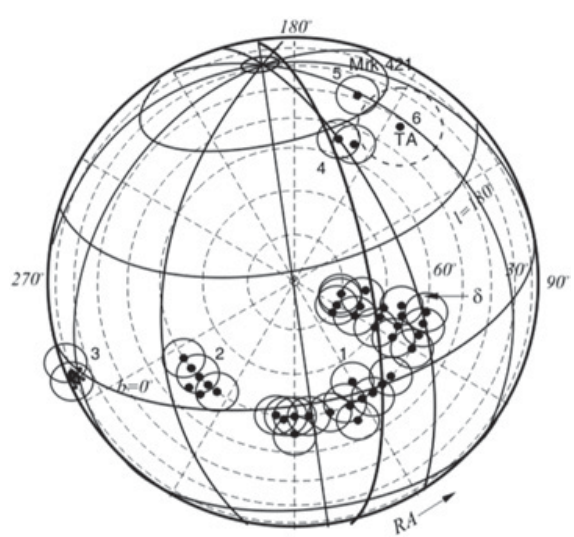

Figure 1. Yakutsk, $E \geq 8.10^{18} \mathrm{eV}$, distribution of particles in the celestial sphere $\bullet$ within a radius $\mathrm{R}<6^{\circ}-\geq 16$ particles were observed. 1, 2, 3, 4 - data of the Yakutsk array; 5 - data of TA around galaxy $\mathrm{Mkr} 421 ; 6-\mathrm{TA}$ data $-\mathrm{R}<20^{\circ}$.

(the Galactic Center), the $4^{\text {th }}$ region -4 . Inside these 6 regions there were $183,11,17,23,14,11$ particles respectively. The chance probabilities to find such a number of events from 938 particles are: $P_{1}(938, \geq 183) \sim$ $6.10^{-5}, \quad P_{2}(938, \geq 11) \sim 0.6, \quad P_{3}(938, \geq 17), \sim 2.10^{-3}$, $P_{4}(938, \geq 13) \sim 0.5, P_{4,5} \sim 0.1$.

Note that the chance probabilities are only significant around the $1^{\text {st }}$ and $3^{\text {rd }}$ groups of pulsars at radius $\mathrm{R}<$ $6^{\circ}$ and are $3 \sigma$ more than expected in the case of isotropy. The maximum number of events $20 \leq n \leq 23$ at $\mathrm{R}<6^{\circ}$ are observed around the $1^{\text {st }}$ groups of pulsars: PSR 0141+6009, 0146+6145, 0147+5922, 0157+6212, $0215+6218$. These pulsars are in the galactic plane and have been considered earlier as possible sources of particles [1].

A flux of particles was observed from the side center of the Galaxy (Fig. 1, number 3). All 14 particles arrive at the Yakutsk array at large zenith angles, $60^{\circ} \geq \theta \geq 38^{\circ}$, and from only one direction as neutral particles of dark matter (the trajectories of these particles are not similar to other particles arriving directly from one direction only - the Galactic Center without any deviation). From 6 showers we have no information about the muon component data - these showers were registered in the summer (in summer muon detectors do not operate

(C) The Authors, published by EDP Sciences. This is an Open Access article distributed under the terms of the Creative Commons Attribution License 4.0 (http://creativecommons.org/licenses/by/4.0/). 
because they are underground in permafrost and possible melting permafrost). In the autumn after repair the muon detectors begin to operate. Note that an excess of particles was found from the direction of the Galactic center mainly in autumn at energy $E \sim 10^{18} \mathrm{eV}$ (according to theory maximum fluxes of particles of dark matter are expected in autumn [2]). We suppose that we registered particles of dark matter. The AGASA and Sydney arrays also observed a flux of particles from the same direction - the side center of the Galaxy $[3,4]$.

\section{Conclusion}

We have found correlations between the arrival directions of ultrahigh energy particles with $E<4 \times 10^{19} \mathrm{eV}$ and the positions of pulsars and an anisotropy of the arrival directions of these particles from the side of the galactic plane for both the Yakutsk array data and the Telescope Array data at energy $E>5.7 \times 10^{19} \mathrm{eV}$ at high galactic latitudes. Our analysis of the data shows that the cosmic rays observed up to $4 \times 10^{19} \mathrm{eV}$ are galactic and above this energy most likely extragalactic.

\section{References}

[1] Mikhailov A.A., Analysis of the Arrival Directions of Ultrahigh Energy Particles. Discovery of Cosmic Rays, book, Denver, June 26-28, 2012, ed. J. Ormes

[2] Kuzmin V.A., Rubakov V.A., Yadernaya Fizika, 1998, 61, 1122

[3] Anda R., Aguirre C., Tachi K., Proc.17-th ICRC, Paris, 1981, 2, 164

[4] Bray A.D., Horton., McCusker C.B.A., Proc.17-th ICRC, 1981, 2, 168 